\title{
Personalising patient care within dentistry
}

\author{
Kishan Patel $^{\star 1}$ and Emma Walshaw ${ }^{1}$
}

\section{Key points}

Reminds readers to shift their focus from treating cases to treating patients.

Investigates the idea of formulating a 'problem list' along with a list of diagnoses aiding patientcentric care.
Encourages clinicians to utilise published evidence focusing on patient-centred outcomes and experiences to better inform their consent process and not just clinical evidence.

\begin{abstract}
Putting patients' interests first should always be at the forefront of a dental practitioner's mind. When treatment planning, it can be possible to lose sight of the patient: their primary concerns, ability to cope with treatment and what they regard success to be. Introducing key concepts such as a 'problem list' and applying the current evidence base for patient-reported outcomes and experience measures can ensure the focus of dental treatment never wavers from holistic patient care. This opinion piece will focus on the current attitudes of dental practitioners and act as a reminder to ensure that their practice is patient-focused at all times.
\end{abstract}

\section{Introduction}

The concept of patient-reported outcomes and experiences is not new within the dental realm, however in daily practice it can possibly be overlooked. The General Dental Council (GDC) have published nine standards for all dental professionals to adhere to. Its first is to 'put patients' interests first'. The Picker Institute $^{1}$ have also developed a set of core principles for patient-centred care, which seconds the GDC standards (Fig. 1). As practitioners, we may be fascinated more by a clinical case, rather than the patient at the end of said case. Consequently, patient outcomes and experiences are somewhat overlooked when providing treatment. Guiding principles for successful treatment should be patientfocused, not case-focused.

'Sheffield, UK.

*Correspondence to: Kishan Patel

Email: kishanpatel15@hotmail.com

Accepted 5 April 2019

DOI: 10.1038/541415-019-0519-9

\section{A problem list?}

The typical patient consultation begins with taking numerous histories from the patient, be that the history of the presenting complaint, the medical history, dental history or social/family history. This, combined with clinical examination and subsequent investigations, will hopefully provide the clinician with sufficient information to list several diagnoses, for which a treatment plan will be devised. All of a sudden, the focus of the consultation moves from patient-focused to case-focused, and key insight into the former may be lost.

In adjunct to developing a list of diagnoses, clinicians should endeavour to produce a 'problem list', with the help of well-established diagnoses. For example, a diagnosis of 'acquired tooth loss' may translate to 'missing tooth upper left first molar tooth' in the 'problem list'. The aim of the 'problem list' is to ensure that diagnoses are patient-specific, and ensures that the all-important patient dimension is not. The benefits of discussing a carefully considered problem list are numerous. It allows for a better rapport to be built with the patient, a more patient-tailored treatment plan to be constructed and, ultimately, a more informed consent process to be developed.

A patient may present with a variety of problems and, upon discussion of said problem list, may only decide to rectify some

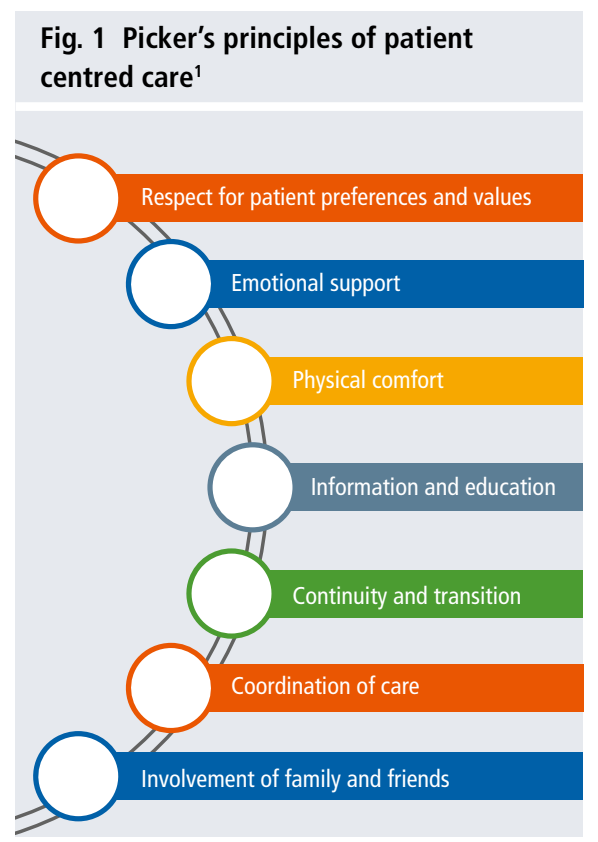


of them. Developing a problem list, along with a list of diagnoses, is well-established in orthodontics. It aids the practitioner to present a variety of treatment options to the patient, with varying levels of interventions and complications. Often, more complex treatment plans will aim to correct all the problems on the list, whereas less complex ones will aim to correct some of the problems, and a compromised outcome is the result. The key with this is the fact that the patient is involved from the outset in the decision-making process. Correcting an underlying skeletal discrepancy may involve orthognathic surgery, however the patient may opt to accept the discrepancy and have treatment resulting in 'orthodontic camouflage'. Such a decision can only be the result of a frank, patient-centric conversation after developing a jargon-free 'problem list'.

\section{Defining success}

Success means different things to different people. Often we try to impart objectivity on to what is usually a subjective matter. As dentists, we are taught and trained to regard success in a binary manner, but it's more complex than that. Periodontal treatment success will undoubtedly include a reduction of bleeding on probing, reduction in sites with suppuration and a reduction in pocket probing depths (PPDs). However, these are all parameters which we as clinicians look for; unfortunately, they will often mean not very much to our patients. The key to a successful periodontal treatment plan is patient motivation and compliance. Their motivating factors may be fear of tooth loss or reduction in halitosis; aspects which we as clinicians find difficult to measure due to our inability to successfully record it on a pre-determined, patient-unspecific 'full periodontal assessment' chart. Therefore, it begs the question, what is success for this patient? A reduction in PPDs, or patient-reported reduction in halitosis and subsequent increased self-confidence? Undoubtedly clinician-specific treatment outcomes and patient-specific ones are inextricably linked and hopefully will align, but surely one must be more important when defining success.

The concept of 'surrogate endpoints' and 'clinical endpoints' is one that is relatively well-established in the medical world. Such terminology was founded to define what the endpoint of various medical trials were; however, these can be extrapolated to the clinical world and help us define success. A clinical endpoint is a patient-centric measure, focusing on how a medical (or dental) encounter affects how the patient feels, functions or survives. This should be the true endpoint and the true aim for any treatment undertaken. A surrogate endpoint is a substitute for a clinical endpoint. These are often easier to measure and easier to quantify, and therefore reported more often than clinical endpoints. Translating this to define the treatment endpoints for the periodontal patient described above, a clinical endpoint would be no future loss of teeth due to periodontal disease and a reduction of halitosis. The surrogate endpoint would be no bleeding on probing, no PPDs over $3 \mathrm{~mm}$ and no suppuration.

Having highlighted the importance of patient-centred success, we must not disregard the surrogate markers for true clinical endpoints. As clinicians, we have completed an undergraduate dental degree to learn and appreciate these. It is, however, our job to translate our surrogate endpoint findings to patient-centred clinical endpoints; that is, it is our job to say something along the lines of:

'Sir/Madam, after undertaking an extensive clinical examination of the status of your gums, you have many sites which bleed and exude pus. What this means for you is that should you not get your gum disease under control, you will continue to have bad breath and have a high chance of losing teeth due to gum disease'.

Most good clinicians will do this anyway, but thinking of a consultation in this way shifts the focus of the examination from the dentition (or the case) to the patient as a whole.

\section{Aesthetics in dentistry}

Access to information and social media has a lot to answer for when it comes to understanding why patients are evermore fixated on their aesthetics. Within the dental arena, patients want an increasing amount of dental treatment to be undertaken to enhance what is an otherwise clinically healthy dentition. Aesthetic treatments such as dental bleaching, diastema closures and limited treatment orthodontics (also known as shortterm orthodontics) are becoming absolutely essential within the generalist's armoury.

The endpoint defining success for any aesthetic treatment is qualified by the patient. What one patient considers aesthetically pleasing will be different from another's.
Extrapolating this, when embarking on an aesthetic course of treatment, whatever that may be from placement of aesthetic veneers to anterior realignment of teeth, the clinical endpoint of success should be discussed with the patient from the outset. The outcome of this conversation should form the basis of treatment objectives and inform a problem list to address via the course of treatment. When patient expectations are fantastical, clinicians need to engage in a two-way dialogue to ensure patients are aware of treatment limitations. Professional ethics should be maintained with discussed aesthetic treatment and, above all, realistic expectations should be emphasised and highlighted. Use of clinical photographs may aid in obtaining valid consent for any proposed aesthetic treatment.

Developing a patient-centred approach for these cases will ensure that upon completion of treatment, patients aren't disappointed with their final outcome. A patient who has anterior crowding may want to embark on a course of short-term orthodontics. The final clinical result may adequately correct labial segment crowding, but consequently the patient's overjet may increase. The resultant latter occlusal trait may be hugely undesirable for the patient, resulting in a potential failure with the course of treatment from a patientspecific, clinical endpoint perspective. Having a thorough discussion of patient desires from aesthetic treatment and fully documenting them through each stage of treatment will hopefully enable alignment of the clinician's aims of treatment with the patient's.

\section{Patient-centric ways to consider the literature}

Evidence-based medicine (and dentistry) concerns itself with marrying the most appropriate evidence within the literature to patients in order to optimise clinical outcomes. The clear majority of clinical evidence presented within the literature concerns itself with clinical outcomes of various interventions. For example, the success rates for resinretained fixed partial dentures (RRFPDs) has been widely reported in terms of debond rates, caries progression and aesthetic considerations, with the five-year survival rate postulated at approximately $80 \% .{ }^{2}$ Similar studies have been conducted to evaluate five-year success rates for dental implant-retained prostheses, deemed to be approximately $95 \% .^{3}$ When considering such evidence, a clinician will be 
better equipped to provide survival rates to a patient presenting with a missing lower left first molar tooth. However, there is another category of evidence which the clinician may consider when evaluating and presenting treatment options: patient-centred ones.

PROMs (patient-reported outcome measures) and PREMs (patient-reported experience measures) are vital, but somewhat overlooked methods of evaluating best treatment options for patients. PROMs are concerned with validated tools used to evaluate patient outcomes to specific treatments. Such parameters to investigate can include oral health-related quality of life (OHRQoL). PREMs are used to explore patient experiences when considering specific treatments and are often delivered through patient questionnaires.

Such studies are vital to consider when outlining treatment options, as it may make all the difference to the patient when making their decision. OHRQoL measures suggest that there is no difference between the provision of a two-unit RRFPD and a single-unit implantsupported prosthesis when replacing a single missing unit. ${ }^{4}$ Providing such patient-centred evidence (in a non-jargon format) may be the pivotal factor in the patient's decision as to what treatment modality to pursue when replacing a single missing posterior unit. Even though five-year survival rates within the literature are higher than that over RRFPDs, the fact that, from a PROMs perspective, there is no significant difference between the treatment modalities, may convince the patient to go for the less invasive, more cost-effective RRFPD option. As you may appreciate, providing patient-based evidence when discussing treatment options allows for a more comprehensive discussion, resulting in a more valid informed consent procedure. A systematic review is currently underway to investigate in more detail the OHRQoL outcomes of two-unit, cantilevered RRFPDs to better inform clinicians and their patients on these issues, ${ }^{5}$ highlighting the importance and topical nature of research into PROMs and PREMs to better the delivery of care we offer to our patients.

\section{Conclusion}

As clinicians, we are forever obsessed with providing the best possible treatment, resulting in the best possible outcomes. However, what we define as the best may not be the best in the patient's perspective, and indeed it is the patient who we are in this profession to best serve. Sometimes a pragmatic approach needs to be taken. There is already a paradigm shift developing within dentistry to humanise it and ensure that it is more patient-centric. This is essential to ensure successful outcomes and patient satisfaction. Clinical expertise should be combined with patient-centred true clinical endpoints to achieve a shared common treatment goal.

\section{References}

1. Gerteis M, Edgman-Levitan S, Daley J, Delbanco T L. Through the patient's eyes: understanding a promoting patient-centred care. San Francisco: Jossey-Bass, 1993.

2. King P A, Foster L V, Yates R J, Newcobe R G, Garrett $M$ J. Survival characteristics of 771 resin-retained bridges at a UK dental teaching hospital. Br Dent J 2015; 218: 423-428.

3. Jung R E, Pjetersson B E, Glauser R, Zembic A, Zwahlen M, Lang N P. A systematic review of the 5 -year survival and complication rates of implant-supported single crowns. Clin Oral Implants Res 2008; 19: 119-130.

4. Lam W Y, McGrath C P, Botelho M G. Impact of complications of single tooth restorations on oral health-related quality of life. Clin Oral Implants Res 2014; 25: 67-73.

5. Hoyle P, Patel K, Benson P. Oral health-related quality of life in patients restored with resin-retained bridges: a systematic review. PROSPERO 2019; CRD42019114427. Available at http://www.crd.york.ac.uk/PROSPERO/ display_record.php?ID=CRD42019114427 (accessed June 2019). 